\title{
"Spurious nonsignificance" with rank correlation: Some alternative methods and a coefficient of general association
}

\author{
EDWARD L. WIKE \\ University of Kansas, Lawrence, Kansas
}

\begin{abstract}
Buckalew and Pearson (1983) termed the failure to detect a nonmonotonic relationship with a rank-order correlation as "spurious nonsignificance." Their hypothetical set of data was found to be nonrandom by a one-sample runs test and then was summarized by two rhos. Three other methods of testing for nonmonotonic relationships-Edgington's $(1961,1969)$ trend test and two graphical tests (runs and point-pairs) of general association by Quenouille $(1952,1959)-$ were described and applied to the Buckalew-Pearson example. Finally, their data were summarized by a sequential correlation coefficient, psi, which is an index of general association. Psi, which was devised by Quenouille (1952), is a nonparametric version of eta.
\end{abstract}

In a recent paper, Buckalew and Pearson (1983) demonstrated the fallacy of applying rank-order correlation, rho or tau, to a nonmonotonic relationship. Their application of rho to a hypothetical set of data, which conformed to an inverted-U relationship, resulted in a nonsignificant rank correlation coefficient of -.043 . To assess the presence of a nonmonotonic association, the authors ranked the two variables, ordered the cases on the $\mathrm{X}$ ranks, subtracted the $\mathrm{Y}$ ranks from the $X$ ranks, and performed a one-sample runs test on the signs of the differences. They found four runs with 6 positive differences $\left(\mathrm{N}_{1}\right)$ and 10 negative differences $\left(\mathrm{N}_{2}\right)$. The significance of the number of runs was evaluated by reference to a section of the Swed-Eisenhart (1943) tables, reprinted in Siegel (1956). After observing a significant $(p=.05)$ nonmonotonic relationship, the authors commented: "Unfortunately, no test for curvilinear rank correlation is readily available, although an interesting possibility emerges" (p. 77). The "interesting possibility" consisted of calculating two independent rhos upon the two data segments that were obtained when the data were dichotomized between the second and third run.

The aims of this article are: (1) to present some alternative methods for testing for nonmonotonic association; and (2) to describe an index of nonmonotonic association, the sequential correlation coefficient, psi, which was developed by Quenouille (1952).

The three alternative nonparametric tests for general association are: (1) a test for nonmonotonic trend proposed by Edgington $(1961,1969)$; (2) a graphical test of general association (Quenouille,

The author's mailing address is: Department of Psychology, University of Kansas, Lawrence, Kansas 66045.
1959); and (3) a point-pairs graphical test of general association (Quenouille, 1952, 1959). Edgington explicated his test by applying it to a set of Weber ratios that had been ordered on $\log$ intensities. As in the Buckalew-Pearson (1983) procedure, the number of runs served as the test statistic (a run was defined as a group of one or more signs). However, the signs in the Edgington test corresponded to those from the successive differences (i.e., $\mathrm{O}_{1}-\mathrm{O}_{2}, \mathrm{O}_{2}-\mathrm{O}_{3}$, etc.) among the adjacent Weber ratios. The number of runs, $r$, and the numbered observations, $O$, were then referred to Edgington's table (Bradley, 1968; Edgington, $1961,1969)$ to evaluate the significance of a nonmonotonic association. In the case of adjacent tied values, one value of a set was retained, the remaining values were discarded, and the number of observations was reduced by the number of discarded values. When this procedure was applied to the $\mathrm{Y}$ scores or the $Y$ ranks of Table 2 of Buckalew and Pearson (1983) after they had been ordered on $X$, the signs of the first differences were: ----+-+++ . From the Edgington table, the probability of obtaining four runs with 10 observations is .0633 .

In Quenouille's (1959) graphical test of general association, a scatter diagram is made of the scores. Then a horizontal medial line is drawn through the scatter diagram so that half of the points fall above the medial line and half fall below. Points falling on the medial line are discarded and the number of points, $\mathrm{N}$, is reduced by the number of discarded points. A run is defined as a set of consecutive points on the same side of the medial line. The number of runs and points, N, are then entered in Quenouille's (1959) Table 7, which is an abbreviated version of Swed and Eisenhart's (1943) Table 1. The reduced table is made possible by the fact that the medial line usually equalizes the two classes of outcomes (i.e., $\mathrm{N}_{1}=\mathrm{N}_{2}$ ). ${ }^{1}$ 
The Quenouille (1959) test has been applied in Figure 1 to the Buckalew-Pearson (1983) data. Since four points fall on the medial line, they are discarded. The first three points below the medial line constitute a run, the next six points above the line constitute a second run, and the last three points below the line constitute a third run. Thus, there were 3 runs, for $N=12$ points. From Quenouille's (1959) Table 7, the probability of this outcome is .05 .

A third method of assessing a general association is Quenouille's $(1952,1959)$ point-pairs test. As in the previous Quenouille method, a medial line is drawn through the scatter diagram and the points on the medial line are discarded. The pairs of adjacent points falling on the same side of the medial line are termed point-pairs. The significance of a general association can then be obtained by entering Table IV of Quenouille (1952, p. 227) or Table 8 of Quenouille (1959) with the number of point-pairs, $M$, and total number of points, $N$. In Figure 1, the point-pairs have been connected by arcs and have been numbered. The probability of observing nine point-pairs with 12 points is .05 from Quenouille's (1959) Table 8. Since the point-pairs table appears only in books that are probably out of print, the table is likely to be inaccessible. Fortunately, Quenouille (1952, p. 227) presented a large-sample formula for calculating the critical numbers of point-pairs. The critical number of point-pairs $=$ the integer next above $[(\mathrm{N}-1) / 2]$ $+\mathrm{Z}(\mathrm{N}-1)^{1 / 2}$, where $\mathrm{Z}=0.823$ for $\alpha=.05$ and 1.163 for $\alpha=.01$. In the present example, $[(12-1) / 2]+$ $0.823(12-1)^{1 / 2}=8.230$. Therefore, when $\mathrm{N}=12$, the critical number of point-pairs is 9 for $\alpha=.05$. It is noteworthy that Quenouille's (1959) point-pairs Table 8 is completely reproducible by the large-sample formula. ${ }^{2}$ In summary, then, two of the three alternative

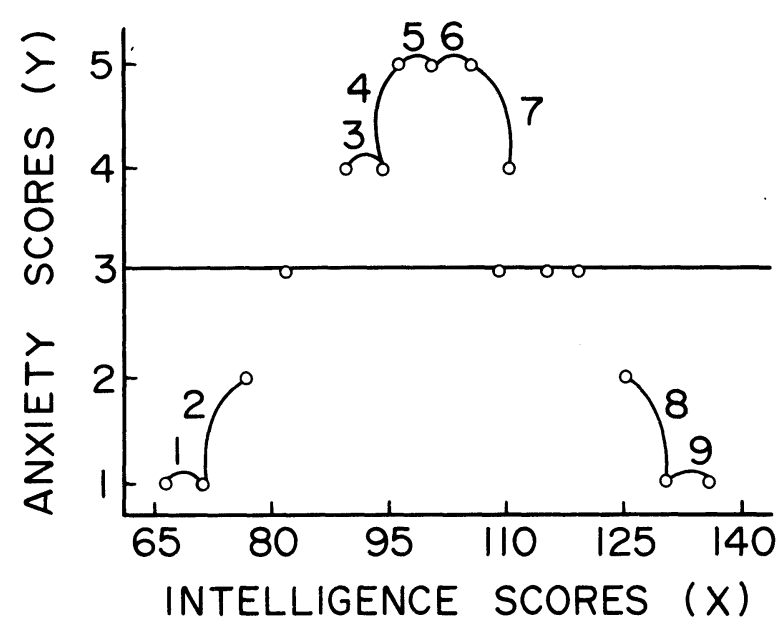

Figure 1. Application of Quenouille's (195?) runs and pointpairs tests to the Buckalew-Pearson example. methods suggest that a significant general association exists in the Buckalew-Pearson (1983) example and the third method barely misses significance at $\alpha=.05$.

Although either the Buckalew-Pearson (1983) onesample runs test or the three methods described here are possible techniques for determining whether or not a significant nonmonotonic association is present, the point-pairs procedure has an advantage. Instead of calculating rank-order correlations on the curve segments as Buckalew and Pearson suggested, it is possible to obtain a single index of general association from the point-pairs test. Regarding this coefficient of sequential correlation, psi, Quenouille (1952), who did pioneering work in time-series analysis, asserted: "The point-pair test is most useful where one variable takes a series of ordered values and the other appears to be nonlinearly dependent upon it. In particular, time-series may be tested for general trend or association between successive observations"' (1952, p. 44). The sequential coefficient, psi, has the following characteristics: its value is +1 for a perfect increasing or decreasing relationship, 0 for unrelated observations, and near +1 for a perfect, but curved, relationship. Thus, psi has the same potential range of values, 0 to +1 , as its parametric counterpart, eta. The formula for the sequential coefficient is: $p s i=[2(M) / N-2]-1$, where $M$ is the number of point-pairs and $\mathrm{N}$ is the number of points. For the Buckalew-Pearson problem, psi $=[2(9) /$ $12-2]-1=.80$. Obviously, this value of the coefficient, which is significant by a runs or point-pairs test, as shown previously, is a more reasonable description of the data than a nonsignificant rho or tau. And, of course, the sequential coefficient is applicable to more complex curvilinear relationships than an inverted-U function. Finally, by comparing the absolute magnitudes of tau and psi on the same set of data, a rough indication can be gained as to the degree of curvature for a relationship (Quenouille, 1952 , p. 46). If tau and psi are quite similar, then the relationship does not depart greatly from monotonicity. If the two coefficients are markedly different, as in the Buckalew-Pearson example, then psi summarizes the nonmonotonic relationship more adequately than does tau.

\section{REFERENCES}

Bradley, J. V. Distribution-free statistical tests. Englewood Cliffs, N.J: Prentice-Hall, 1968.

Buckalew, L. W., \& Pearson, W. H. "Spurious nonsignificance" in rank correlation. Bulletin of the Psychonomic Society, 1983, 21, 76-78.

Conover, W. J. Practical nonparametric statistics. New York: Wiley, 1971.

Edgington, E. S. Probability table for number of runs of signs of first differences in ordered series. Journal of the American Statistical Association, 1961, 56, 156-159. 
Edgington, E. S. Statistical inference: The distribution-free approach. New York: McGraw-Hill, 1969.

Quenouille, M. H. Associated measurements. London: Butterworths, 1952.

Quenouille, M. H. Rapid statistical calculations. New York: Hafner, 1959.

Siegel, S. Nonparametric statistics for the behavioral sciences. New York: McGraw-Hill, 1956.

Swed, F. S., \& Eisenhart, C. Tables for testing randomness of grouping in a sequence of alternatives. Annals of Mathematical Statistics, 1943, 14, 66-87.

\section{NOTES}

1. More extensive tables are presented in Bradley $(1968$, p. 362) and Conover (1971, p. 414). Bradley's table also includes the critical numbers of runs for $\alpha=.01$. The Conover table has a range of $\alpha$ levels for both too few runs and too many runs, but fewer $\mathrm{N}_{1}$ and $\mathrm{N}_{2}$ values.

2. Quenouille's formula is used to calculate the required numbers of point-pairs for even values of $\mathrm{N}$. The same number of point-pairs is required for the next odd N. For example, when $\mathrm{N}=12$ or 13, the critical number of point-pairs is 9 for $\alpha=.05$.

(Manuscript received for publication June 12, 1983.) 